\title{
Schedule-dependent synergistic effect of rituximab on methotrexate chemotherapy against lymphoma of the central nervous system
}

\author{
JUYOUN JIN ${ }^{1,2^{*}}$, KYEUNG MIN JOO ${ }^{2,5^{*}}$, YOONHEE NAM ${ }^{6}$, DAE HYUN KIM ${ }^{7}$, SE JEONG LEE ${ }^{1,2}$, \\ MI-YOUNG JO ${ }^{1,2}$, YOUNGGEON JIN ${ }^{1,2}$, HYEONG-SEOK KIM ${ }^{1,2}$, SOO WON SEO ${ }^{4}$, \\ SEOK JIN KIM ${ }^{3}$, DO-HYUN NAM ${ }^{1,2 * *}$ and WON SEOG KIM ${ }^{3 * *}$ \\ ${ }^{1}$ Department of Neurosurgery, ${ }^{2}$ Cancer Stem Cell Research Center, ${ }^{3}$ Department of Hematology-Oncology, \\ ${ }^{4}$ Department of Biomedical Engineering, Samsung Medical Center and Samsung Biomedical Research Institute, \\ Sungkyunkwan University School of Medicine, Gangnam-gu, Seoul 135-710; ${ }^{5}$ Department of Anatomy, \\ Seoul National University College of Medicine, Jongno-gu, Seoul 110-799; ${ }^{6}$ Gwacheon Foreign Language \\ High School, Gwacheon-si, Gyeonggi-do 427-805; ${ }^{7}$ Korean Minjok Leadership Academy, \\ Anheung-myeon, Hoengseong-gun, Gangwon-do 225-823, Korea
}

Received June 28, 2010; Accepted August 20, 2010

DOI: $10.3892 / \mathrm{etm} .2010 .143$

\begin{abstract}
We hypothesized that methotrexate (MTX) normalizes the increased permeability of the blood-tumor barrier and thus reduces the accessibility of rituximab (RTX) to central nervous system (CNS) lymphoma. Here, we evaluated the combinational treatment capability of RTX and MTX using an alternative treatment schedule against CNS lymphoma. We developed a CNS lymphoma animal model that closely mimics the morphological and molecular characteristics of human CNS lymphoma by injecting Raji human Burkitt lymphoma cells into the brains of immune-compromised mice and tested a novel combinational treatment schedule by which penetration of RTX was not influenced by MTX administration. RTX was conjugated with Alexa Fluor 680, and its distribution in the brain was analyzed by in vivo imaging. When MTX treatment
\end{abstract}

Correspondence to: Dr Do-Hyun Nam, Department of Neurosurgery, Samsung Medical Center and Biomedical Research Institute, Sungkyunkwan University School of Medicine, Gangnam-gu, Seoul 135-710, Korea

E-mail: nsnam@skku.edu

Dr Won Seog Kim, Department of Hematology-Oncology, Samsung Medical Center and Biomedical Research Institute, Sungkyunkwan University School of Medicine, Gangnam-gu, Seoul 135-710, Korea E-mail:wskimsmc@skku.edu

${ }^{*}$ Contributed equally

*** Joint senior authors

Key words: central nervous system lymphoma, methotrexate, rituximab, combination therapy, mouse model was followed by a 3-day post RTX administration, RTX was scarcely distributed in the brain, and there were only modest statistically insignificant therapeutic effects compared with the control mice which received sham injections. In contrast, RTX administration followed by a 3-day post MTX treatment showed significantly increased distribution of RTX and significantly reduced tumor volume in the brain. Collectively, our data demonstrate that RTX can be successfully combined with MTX using an alternative treatment schedule that allows increased distribution of RTX in CNS lymphoma.

\section{Introduction}

Rituximab (RTX), a monoclonal antibody targeting the B-cell-specific CD20 antigen, is an effective treatment agent for B-cell non-Hodgkin's lymphoma (NHL) $(1,2)$. However, its application for the treatment of central nervous system (CNS) lymphoma is still controversial since the blood-brain barrier (BBB) is relatively impermeable to antibodies with high molecular weights $(3,4)$. Conversely, RTX could exhibit therapeutic effects on CNS lymphoma when the permeability of the blood-tumor barrier increases.

The primary therapeutic option for CNS lymphoma is intravenous high-dose methotrexate (MTX)-based chemotherapy (5-7). Previously, RTX and temozolomide showed significant therapeutic effects against relapsed primary CNS lymphoma $(8,9)$. However, there are no indisputable data showing successful combination of MTX with RTX. We hypothesized that MTX normalizes the increased permeability of the bloodtumor barrier and thus reduces the accessibility of RTX to CNS lymphoma (10). In the present study, we administered RTX to a CNS lymphoma animal model prior to MTX treatment to avoid the normalizing effects of MTX on the blood-tumor barrier, and our novel treatment modality conferred synergistic therapeutic effects against CNS lymphoma. 


\section{Materials and methods}

Cell culture and reagents. Raji human Burkitt lymphoma cells (American Type Culture Collection, Manassas, VA) were grown in RPMI-1640 (Gibco BRL, Grand Island, NY) supplemented with $10 \%$ FBS, 2 mM L-glutamine, penicillin $(100 \mathrm{U} / \mathrm{ml})$, and streptomycin $(100 \mu \mathrm{g} / \mathrm{ml})$. MTX and RTX (Mabthera) were purchased from Mayne Pharmaceuticals and Roche Pharmaceuticals, respectively.

Primary CNS lymphoma animal model. Anesthetized 6-week-old male Balb/c-nu mice were secured in a rodent stereotactic frame, a hollow guide screw was implanted into a small drill hole made $2 \mathrm{~mm}$ right and $1 \mathrm{~mm}$ anterior to the bregma, and $5 \times 10^{4}, 5 \times 10^{5}$ and $5 \times 10^{6}$ Raji cells in $5 \mu$ HBSS were injected through this guide screw into the white matter at a depth of $3 \mathrm{~mm}$ [anterior/posterior (AP) $+1.0 \mathrm{~mm}$, medial/ lateral (ML) $-1.7 \mathrm{~mm}$, dorsal/ventral (DV) $-3.2 \mathrm{~mm}$ ]. All of the experiments where mice received antitumor therapy were performed using an inoculum of $5 \times 10^{5}$ Raji cells. Animal experiments were approved by the appropriate Institutional Review Boards of the Samsung Medical Center, Seoul, Korea and conducted in accordance with the 'National Institute of Health Guide for the Care and Use of Laboratory Animals' (NIH publication no. 80-23, revised in 1996).

Evaluation of antitumor activity. Twelve days after the intracerebral injections of Raji cells, mice were randomly assigned to receive sham treatment (Group A), MTX administration followed by RTX treatment (Group B), or RTX administration followed by MTX treatment (Group C) (Fig. 3A). Twenty-one days after the injection of Raji cells, the mice were sacrificed. Brains were harvested and processed for paraffin embedding.

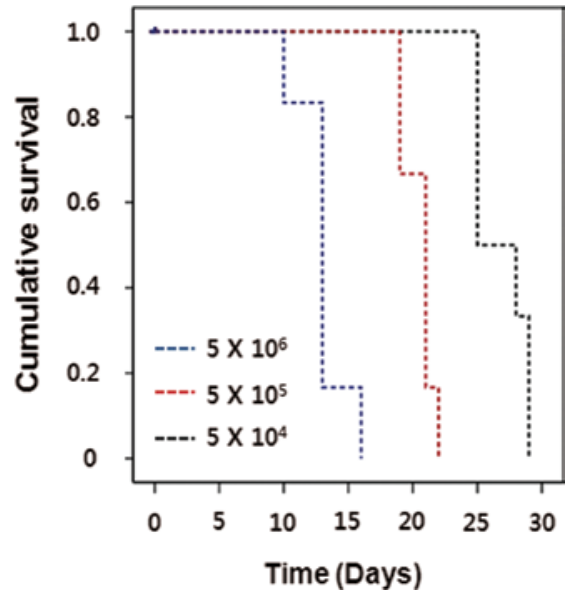

Figure 1. Survival curves of the nude mouse CNS lymphoma model. Nude mice were injected with lymphoma cells $\left(0.5,5\right.$, and $50 \times 10^{5}$ cells/mouse $)$ and sacrificed upon development of neurological symptoms or a $25 \%$ weight loss.

The tumor volume was calculated by measuring the section with the largest tumor portion applying the formula: (width) ${ }^{2}$ $\mathrm{x}$ length $\mathrm{x} 0.5$.

In vivo quantification of the penetration of RTX into the CNS lymphoma. To evaluate the penetration of RTX into the mouse brains bearing CNS lymphoma, RTX conjugated with Alexa Fluor 680 (RTX-AF680) was utilized. RTX was conjugated with Alexa Fluor 680 according to the manufacturer's protocol (SAIVI Alexa Fluor 680 antibody/protein $1 \mathrm{mg}$ labeling kit; Invitrogen, CA, USA). Three days after i.v. injection of RTX-AF680 (Fig. 3A), mice were anesthetized with 2-3\% isoflurane. The signal from RTX-AF680 was detected in the

A
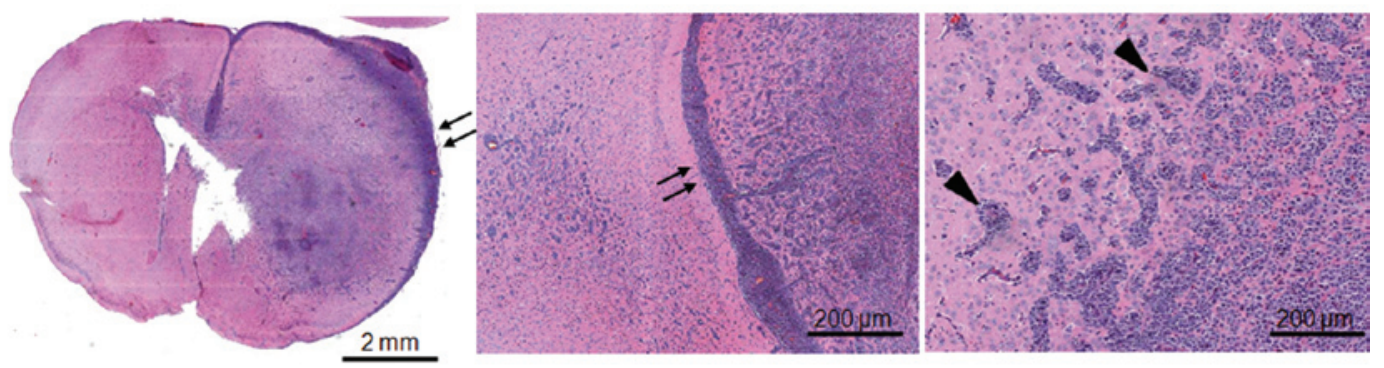

B

C

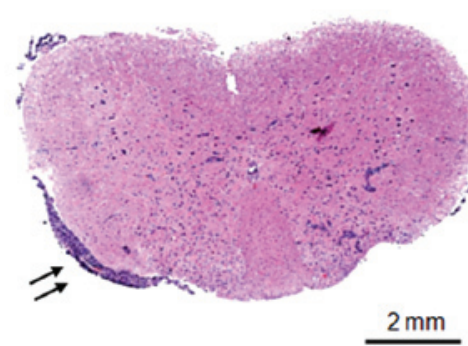

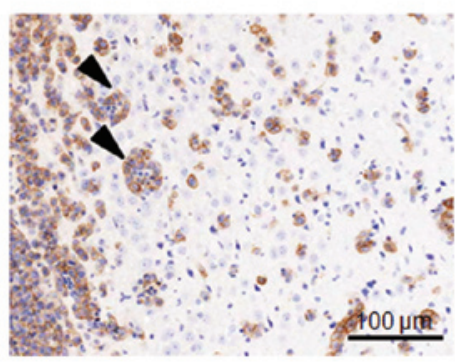

D

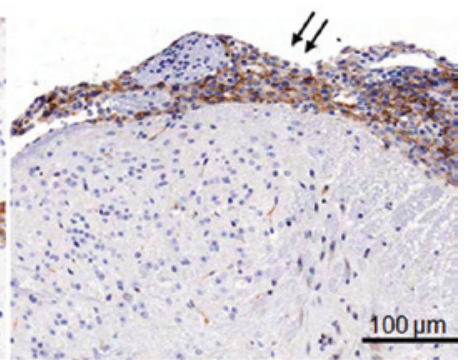

Figure 2. CNS lymphoma animal model. Brain tumors derived from implanted Raji lymphoma cells were obtained for pathologic analysis 21 days after tumor cell implantation. Sections were stained with H\&E (A and B), or immunohistochemistry against CD20 was performed (C and D). (A) Lymphoma cells were mainly localized in the injected hemisphere and invaded the contralateral hemisphere (arrows, leptomeningeal seeding; arrow heads, perivascular cuffing). (B) Lymphoma cells were also observed in the leptomeninges of the spinal cord (arrows, leptomeningeal seeding). Lymphoma cells from the perivascular cuffing in the brain (C) and leptomeningeal seeding in the spinal cord (D) expressed high levels of CD20. 
A
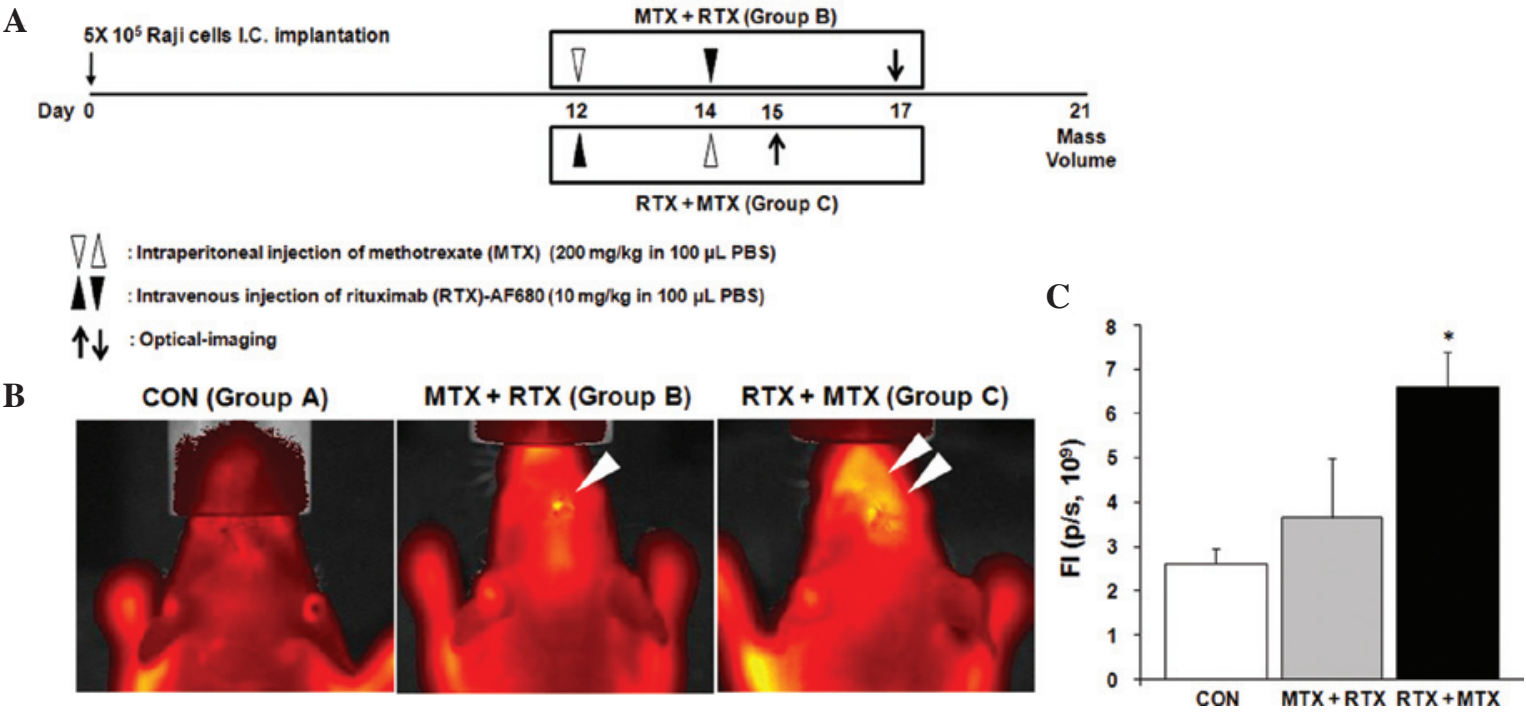

Figure 3. Effects of MTX treatment on the RTX penetration across the blood-tumor barrier. (A) Detailed experimental schedules using the CNS lymphoma animal model are illustrated. (B) Distribution of AF680-labeled RTX in brains bearing lymphoma cells was visualized by in vivo fluorescent optical imaging 3 days after RTX administration. Group A $(\mathrm{CON})$, Sham injection of PBS (Day 12, 14); Group B, (MTX + RTX) = MTX (Day 12) + RTX (Day 14); and Group C $($ RTX + MTX) = MTX (Day 12) + RTX (Day 14). $n=5$ for each group. (C) Fluorescence intensity (FI) was analyzed and compared. Mean \pm SE. ${ }^{*} \mathrm{P}<0.05$ vs. Group A or B.

A

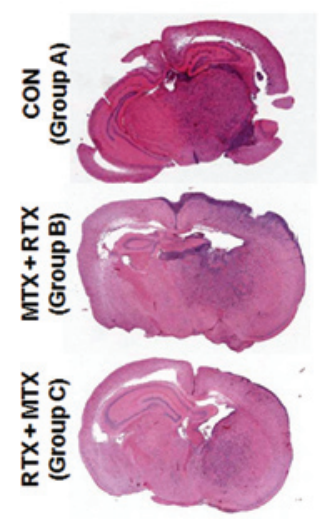

B

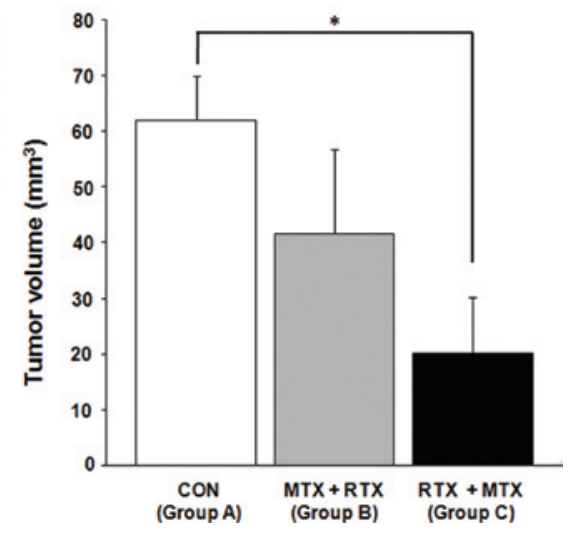

Figure 4. Therapeutic effects of RTX and MTX combination treatments on CNS lymphoma. (A) Brain tumors derived from implanted Raji lymphoma cells were obtained 21 days after tumor cell implantation. Sections were stained with H\&E. (B) The average tumor volumes of each group ( $\mathrm{n}=5$ for each group) were determined and compared. Group A (CON), Sham injection of PBS (Day 12, 14); Group B, (MTX + RTX) = MTX (Day 12) + RTX (Day 14); and Group C, $($ RTX + MTX) = MTX (Day 12) + RTX (Day 14). Mean \pm SE. ${ }^{*} \mathrm{P}<0.05$ vs. CON.

region of the brain using a prototype Xenogen IVIS ${ }^{\circledast}$ Spectrum in vivo imaging system (Caliper Life Science). Fluorescence intensity was analyzed as photons per second $(\mathrm{p} / \mathrm{s})$ by Living Image 3.1 software (Caliper Life Science).

Statistical analyses. Statistical comparisons of the RTX penetration and tumor regression between groups were analyzed by one-way analysis of variance (ANOVA) followed by the least significant difference (LSD) test. All data were presented as means \pm SEM. A values of $\mathrm{P}<0.05$ was considered statistically significant.

\section{Results}

CNS lymphoma animal model. To establish the CNS lymphoma animal model, Raji human Burkitt lymphoma cells were stereotactically injected into the brains of immune compromised Balb/c-nu mice with a range of cell doses $(0.5$, 5 and $50 \times 10^{5}$ cells/mouse), and mice were observed for the development of irreversible neurological symptoms before euthanization. Nude mouse survival times were dependent on the cell dose (Fig. 1). The optimal cell dose of Raji cells was determined by the length of survival. A cell dose of $5 \times 10^{5}$ resulted in $100 \%$ mortality within 21 days (19-23 days) after implantation and was used in all of the subsequent in vivo experiments. Huge infiltrative intracerebral tumors were observed in the brain three weeks after the tumor cell injection (Fig. 2A). Pathologic characteristics of CNS lymphoma such as extensive leptomeningeal seeding (arrows in Fig. 2A) and perivascular cuffing at the periphery of tumors (arrow heads in Fig. 2A) were obvious. In addition to cerebral involvement, lymphoma cells were clearly observed in the leptomeninges of the spinal cord but not in the parenchyma (arrows in Fig. 2B and D, thoracic spinal cord). Most lymphoma cells highly expressed CD20, a B-cell marker (Fig. 2C for perivascular cuffing in the brain, Fig. 2D for leptomeningeal seeding in the spinal cord).

Effects of MTX treatment on the RTX penetration across the blood-tumor barrier. To evaluate our hypothesis, we administrated MTX and RTX to the mice bearing lymphoma cells in their brains according to different schedules (Fig. 3A). Administered RTX was labeled with AF680 to visualize the distribution in the brain in vivo. Each mouse was examined 3 days after RTX treatment (Group B; Day 17, Group C; Day 15), while mice from Group A were examined twice at Day 15 and 17 (Fig. 3A). We did not observe fluorescent 
signals from the brains of the mice not administered RTX (Group A, Fig. 3B). When mice received MTX treatment followed by RTX (Group B), RTX was not accumulated in the brain (Group B, Fig. 3B). In contrast, penetration of RTX across the $\mathrm{BBB}$ or the blood-tumor barrier was significantly increased by changing the treatment order from MTX + RTX to RTX + MTX (Group C, Fig. 3B and C).

Therapeutic effects of RTX and MTX combination treatments on CNS lymphoma. Next, we analyzed whether RTX penetration is associated with therapeutic effects by measuring tumor volumes at Day 21 (Fig. 3A). The RTX and MTX combination treatment, in which MTX administration was followed by RTX injection (Group B), reduced tumor volume by $32 \%$, but this was not statistically significant (Fig. 4A and B; Group A, $62 \pm 8.0 \mathrm{~mm}^{3}$; Group B, $41.7 \pm 15.1 \mathrm{~mm}^{3}$ ). In contrast, mice that received RTX treatment followed by MTX administration showed a significantly reduced tumor volume (Fig. 4A and B; Group C, $20.1 \pm 10.2 \mathrm{~mm}^{3}$, $68 \%$ tumor volume reduction; $\mathrm{P}<0.05)$.

\section{Discussion}

The investigation of CNS lymphoma treatment, biology, pathophysiology, and pathology in humans is troublesome, mainly due to the rarity of the disorder. Our data demonstrated that the intracerebral implantation of Raji cells into immunecompromised mouse brain provides an animal model that closely mimics the morphological and molecular characteristics of human CNS lymphoma (11). Here, we demonstrated that RTX can be successfully combined with MTX using an alternative treatment schedule that allows increased distribution of RTX in CNS lymphoma.

The BBB is relatively impermeable to high molecular weight antibodies $(3,4)$. However, the blood-tumor barrier of brain tumors is more permissive to the penetration of antibodies into tumors, which would enable the application of therapeutic antibodies to brain tumors $(12,13)$. We hypothesized that prior MTX treatment reverses the permissive characteristics of the tumor-blood barrier as there are massive tumor cell apoptosis and normalization of brain tissue structure caused by MTX. By in vivo imaging of RTX distribution, we provided data showing that the treatment schedule significantly alters the accessibility of therapeutic antibodies to brain tumors.

Due to the different mechanisms of action of MTX and RTX, a synergistic effect with improved response rate could be postulated by the combination. However, previous studies have failed to demonstrate the superiority of MTX and RTX combination treatment over MTX or RTX monotherapy (10). In the present study, we demonstrated that MTX influences the penetration of RTX across the blood-tumor barrier, which consequently abolishes the expected synergistic effects. In addition, we developed a novel combination method in which RTX treatment was followed by MTX administration. By applying the altered combination therapy, both accumulation of RTX in the brains bearing CNS lymphoma and strengthening of therapeutic effects of MTX chemotherapy on CNS lymphoma were achieved.
We report the successful combination of MTX and RTX for the treatment of CNS lymphoma in the translational setting. Clinical relevance of this strategy needs to be further elucidated through clinical trials.

\section{Acknowledgements}

This study was supported by a grant of the Korea Healthcare Technology R\&D Project, Ministry for Health and Welfare Affairs, Republic of Korea (A092255) (D.-H. Nam) and the Ministry of Health and Welfare of Korea (0405-MNO1-06040007) (S.W. Seo).

\section{References}

1. McLaughlin P, Grillo-Lopez AJ, Link BK, et al: Rituximab chimeric anti-CD20 monoclonal antibody therapy for relapsed indolent lymphoma: half of patients respond to a four-dose treatment program. J Clin Oncol 16: 2825-2833, 1998.

2. Maloney DG, Grillo-Lopez AJ, Bodkin DJ, et al: IDEC-C2B8: results of a phase I multiple-dose trial in patients with relapsed non-Hodgkin's lymphoma. J Clin Oncol 15: 3266-3274, 1997.

3. Neuwelt EA, Barnett PA, McCormick CI, Remsen LG, Kroll RA and Sexton G: Differential permeability of a human brain tumor xenograft in the nude rat: impact of tumor size and method of administration on optimizing delivery of biologically diverse agents. Clin Cancer Res 4: 1549-1555, 1998.

4. Neuwelt EA: Mechanisms of disease: the blood-brain barrier. Neurosurgery 54: 131-142, 2004.

5. Herrlinger U, Kuker W, Uhl M, et al: NOA-03 trial of high-dose methotrexate in primary central nervous system lymphoma: final report. Ann Neurol 57: 843-847, 2005.

6. Abrey LE, Yahalom J and DeAngelis LM: Treatment for primary CNS lymphoma: the next step. J Clin Oncol 18: 3144-3150, 2000.

7. DeAngelis LM, Seiferheld W, Schold SC, Fisher B and Schultz CJ: Combination chemotherapy and radiotherapy for primary central nervous system lymphoma: Radiation Therapy Oncology Group Study 93-10. J Clin Oncol 20: 4643-4648, 2002.

8. Enting RH, Demopoulos A, DeAngelis LM and Abrey LE: Salvage therapy for primary CNS lymphoma with a combination of rituximab and temozolomide. Neurology 63: 901-903, 2004.

9. Wong ET, Tishler R, Barron L and Wu JK: Immunochemotherapy with rituximab and temozolomide for central nervous system lymphomas. Cancer 101: 139-145, 2004.

10. Jahnke K, Muldoon LL, Varallyay CG, et al: Efficacy and MRI of rituximab and methotrexate treatment in a nude rat model of CNS lymphoma. Neuro Oncol 11: 503-513, 2009.

11. Wang W, Kardosh A, Su YS, Schonthal AH and Chen TC: Efficacy of celecoxib in the treatment of CNS lymphomas: an in vivo model. Neurosurg Focus 21: E14, 2006.

12. Barnett PA, Roman-Goldstein S, Ramsey F, et al: Differential permeability and quantitative MR imaging of a human lung carcinoma brain xenograft in the nude rat. Am J Pathol 146: 436-449, 1995.

13. Neuwelt EA, Barnett PA, Hellstrom KE, Hellstrom I, McCormick CI and Ramsey FL: Effect of blood-brain barrier disruption on intact and fragmented monoclonal antibody localization in intracerebral lung carcinoma xenografts. J Nucl Med 35: 1831-1841, 1994. 